\title{
Comparative genomics of the genus Bifidobacterium
}

\begin{abstract}
Correspondence
Marco Ventura

marco.ventura@unipr.it

Douwe van Sinderen

d.vansinderen@ucc.ie
\end{abstract}

Received 4 March 2010

Revised 30 June 2010

Accepted 6 July 2010

\author{
Francesca Bottacini, ${ }^{1}, 2$ Duccio Medini, ${ }^{3}$ Angelo Pavesi, ${ }^{1}$ \\ Francesca Turroni, ${ }^{1}$ Elena Foroni, ${ }^{1}$ David Riley, ${ }^{4}$ Vanessa Giubellini, ${ }^{1}$ \\ Hervé Tettelin, ${ }^{4}$ Douwe van Sinderen ${ }^{2}$ and Marco Ventura ${ }^{1}$ \\ ${ }^{1}$ Laboratory of Probiogenomics, Department of Genetics, Biology of Microorganisms, \\ Anthropology and Evolution, University of Parma, Italy \\ ${ }^{2}$ Alimentary Pharmabiotic Centre and Department of Microbiology, Bioscience Institute, \\ National University of Ireland, Western Road, Cork, Ireland \\ ${ }^{3}$ Novartis Vaccines and Diagnostics, Siena, Italy \\ ${ }^{4}$ Institute for Genome Sciences, Department of Microbiology and Immunology, \\ University of Maryland School of Medicine, Baltimore, USA
}

\section{INTRODUCTION}

Bifidobacteria are commonly found as part of the microbiota in the human gastrointestinal tract (GIT) (Ventura et al., 2007), where their presence has been positively correlated with the health status of their host (Ventura et al., 2009a, 2009b). Bifidobacteria have been claimed to elicit several health-promoting or probiotic effects, such as strengthening of the intestinal barrier, modulation of the immune response and exclusion of pathogens (Marco et al., 2006; O'Hara \& Shanahan, 2007). Although there is some evidence to support each of these functional claims for particular bifidobacterial strains, the molecular

Abbreviations: COG, clustered orthologous group; GIT, gastrointestinal tract; HGT, horizontal gene transfer; IS, insertion sequence; TUG, truly unique gene.

One supplementary figure and five supplementary tables are available with the online version of this paper. mechanisms by which these activities are achieved remain largely unknown.

In recent years, genome sequencing of bifidobacteria and other health-promoting (or probiotic) bacterial strains has come to the fore, even leading to the designation of a novel discipline named probiogenomics (Ventura et al., 2007, 2009a, 2009b), which aims to improve our knowledge of the diversity and evolution of commensal bacteria of the GIT and to unravel the molecular basis for their presumed health-promoting activities.

Of the currently recognized 34 species of the genus Bifidobacterium, just nine bifidobacterial genomes (representing five species) have been fully sequenced (Barrangou et al., 2009; Kim et al., 2009; Lee et al., 2008; Schell et al., 2002; Sela et al., 2008; Ventura et al., 2009a, 2009b), with another 17 whose genome sequences are still unfinished (NCBI). Notably, for a small number of cases, such as 
Bifidobacterium longum subsp. longum (Lee et al., 2008; Schell et al., 2002) and Bifidobacterium animalis subsp. lactis (Barrangou et al., 2009; Kim et al., 2009), two or more genome sequences are available.

Bifidobacterial genomes range in size from 2.0 to $2.8 \mathrm{Mbp}$ and possess a large number of tRNA molecules (e.g. from 54 to 58), while displaying common architectural features of a typical bacterial chromosome (Ventura et al., 2007). B. animalis subsp. lactis DSM10140 and Bifidobacterium adolescentis ATCC15703 possess the smallest chromosomes, with respective sizes of 2.0 and 2.1 Mbp (Barrangou et al., 2009; Kim et al., 2009). It should be pointed out that these latter bacteria are extensively used in commercial preparations (also known as probiotic foods), and they may therefore have been subjected to growth in synthetic media for an extended number of generations. Such a practice has been demonstrated to cause genome decay in bifidobacteria (Lee et al., 2008), with the loss of chromosomal regions that are apparently dispensable in an environment different from the original ecological niche. Many bacteria contain extrachromosomal elements in the form of plasmids and among the strains that belong to the $B$. longum phylogenetic species, B. longum subsp. longum DJO10A was shown to harbour two plasmids, pDOJH10L (10 kb) and pDOJH10S (3.6 kb), while B. longum subsp. longum NCC2705 possesses a single plasmid, pBLO1 (3.6 kb). The other sequenced genomes appear to lack plasmids, although extrachromosomal elements are commonly identified among bifidobacteria (Alvarez-Martín et al., 2007; Cronin et al., 2007; Lee \& O'Sullivan, 2006; Sangrador-Vegas et al., 2007). There are no obvious unusual species-specific features with regard to the coding density, tRNA gene number or rRNA operons. The number of rRNA loci ranges from two to five (Barrangou et al., 2009; Candela et al., 2004; Kim et al., 2009; Lee et al., 2008; Schell et al., 2002; Sela et al., 2008; Ventura et al., 2009 a, 2009b), which is more variable than the number of rRNA loci identified in other human gut-associated bacteria such as lactobacilli (Makarova et al., 2006). The number of rRNA operons has been claimed to be positively correlated with a micro-organism's ability to colonize and survive in a particular ecological niche (Klappenbach et al., 2000). Thus, the variable number of rRNA loci identified in bifidobacterial genomes suggests that different survival strategies may have been followed.

Among those elements that are considered to belong to the mobilome, i.e. insertion sequence (IS) elements, transposons and prophages, the highest number of IS elements has been identified in the genome of B. longum subsp. infantis ATCC15697 (Sela et al., 2008), whereas a very limited number of such elements were found in the genome of the human oral cavity inhabitant, Bifidobacterium dentium $\mathrm{Bd} 1$ (Ventura et al., 2009a, 2009b).

Thus far, the genome sequence of one or two strains for each of the above-mentioned species has provided information about genome variability at intraspecific level (Barrangou et al., 2009; Kim et al., 2009; Lee et al., 2008; Schell et al.,
2002). However, the question as to how many genomes are necessary to fully describe a bifidobacterial species or the genus Bifidobacterium has not yet been answered.

Bacterial genome data have also been used in order to investigate bacterial evolution through the identification of genes (constituting the core genome) that appear to be conserved among bacteria. Efforts have also been placed on the investigation of the bacterial pan-genome, which represents the core genome containing genes present in a particular taxonomic unit plus the variable elements of a genome. These variable elements are composed of genes that are absent from the genome of at least one member of a taxonomic unit or genes that are unique to a single member of that taxonomic unit (Bentley, 2009; Lapierre \& Gogarten, 2009; Rasko et al., 2008; Tettelin et al., 2005). The pan-genome sizes and rates of recombination have been suggested to reflect differences in niche and lifestyle of the different species (Bentley, 2009).

In this report, we describe a genomic comparison of bifidobacterial species in order to highlight the genome structure complexity of this group of micro-organisms, as well as to provide a novel genomic perspective on the evolution of bifidobacteria.

\section{METHODS}

Genome sequences. The genomes analysed consisted of the nine complete and publicly available Bifidobacterium genomes described in Table 1. Functional annotations including clustered orthologous group (COG) categories were obtained from NCBI.

Pan-genome analysis. The genome comparisons for the pangenome analysis were performed on a larger dataset of 14 publicly available Bifidobacterium genomes, adding five incomplete genome sequences to the nine complete genome sequences (Table 1). Genomic comparisons were performed according to a previously described approach (Tettelin et al., 2005), with the following modifications. Each pair of genome sequences was compared by means of i) wu-BLASTP searches (all protein vs all protein sequences) and ii) wu-tblastn searches (all proteins vs translated genome sequences) (http://www. advbiocomp.com/blast.html). Results from these two comparative searches were then combined in order to minimize the number of false negative matches. Hits were filtered such that homologues were defined as having $50 \%$ sequence similarity over at least $50 \%$ of the length of the protein (Tettelin et al., 2005).

The sequential inclusion of up to 14 strains was then simulated in all possible combinations to determine the number of core and dispensable strain-specific and species-specific genes for each set of sequentially added genome data, after which the generated distributions of values were analysed. Means and the interquartile ranges were used as point estimates of the central tendency and confidence intervals, respectively, for the regression analysis.

The regression analysis for the core genome was performed by fitting a double exponential decay to the data with a weighted least square regression.

$n_{\text {core }}=k_{1} \exp \left(\frac{-N}{\tau_{1}}\right)+k_{2} \exp \left(\frac{-N}{\tau_{2}}\right)+\Theta$

Where, $n_{\text {core }}$ is the average of core gene distributions, $N$ is the number of genomes, $k_{1}, \tau_{1}, k_{2}, \tau_{2}$ and $\Theta$ are free parameters and the inverse 
Table 1. General genome features of Bifidobacterium species included in this study

ND, Not determined; C, complete; UC, incomplete.

\begin{tabular}{|c|c|c|c|c|c|c|c|c|c|}
\hline Species & $\begin{array}{l}\text { Genome } \\
\text { size (bp) }\end{array}$ & $\begin{array}{c}\mathrm{G}+\mathrm{C} \text { content } \\
(\mathrm{mol} \%)\end{array}$ & $\begin{array}{c}\text { No. } \\
\text { genes }\end{array}$ & $\begin{array}{l}\text { No. tRNA } \\
\text { genes }\end{array}$ & $\begin{array}{l}\text { No. } \\
\text { rRNA loci }\end{array}$ & $\begin{array}{c}\text { No. IS } \\
\text { elements }\end{array}$ & Origin & $\begin{array}{c}\text { GenBank } \\
\text { accession no. }\end{array}$ & Status \\
\hline B. adolescentis ATCC 15703 & 2089645 & 60 & 1631 & 54 & 5 & 5 & Human GIT & NC_008618 & C \\
\hline $\begin{array}{l}\text { B. animalis subsp. lactis } \\
\text { Bl-04 }\end{array}$ & 1938709 & 60 & 1631 & 52 & 4 & 6 & Infant faeces & NC_012814 & $\mathrm{C}$ \\
\hline B. dentium $\mathrm{Bd} 1$ & 2636368 & 59 & 2278 & 55 & 4 & 7 & Dental caries & NC_013714 & C \\
\hline $\begin{array}{l}\text { B. longum subsp. infantis } \\
\text { ATCC15697 }\end{array}$ & 2832748 & 60 & 2498 & 79 & 4 & 26 & Infant faeces & NC_011593 & C \\
\hline $\begin{array}{l}\text { B. longum subsp. longum } \\
\text { DJO10A }\end{array}$ & 2375792 & 60 & 1990 & 58 & 4 & 44 & Human GIT & NC_010816 & C \\
\hline B. gallicum DSM20093 & 2019802 & 57 & 1980 & ND & ND & ND & Human GIT & $\mathrm{ABXB} 00000000$ & UC \\
\hline $\begin{array}{l}\text { B. pseudocatenulatum } \\
\text { DSM } 20438\end{array}$ & 2304808 & 56 & 2151 & ND & $\mathrm{ND}$ & $\mathrm{ND}$ & Human GIT & NZ_ABXX00000000 & UC \\
\hline B. catenulatum DSM16992 & 2058429 & 56 & 2011 & ND & ND & ND & Human GIT & NZ_ABXY00000000 & UC \\
\hline B. bifidum NCIM41171 & 2186140 & 62 & 1811 & ND & ND & $\mathrm{ND}$ & Human GIT & NZ_ABQP00000000 & UC \\
\hline B. angulatum DSM20098 & 2007108 & 59 & 1811 & $\mathrm{ND}$ & ND & ND & Human GIT & NZ_ABYS00000000 & UC \\
\hline
\end{tabular}

square of the interquartile ranges of core genome distributions as weights. The best fit was obtained with $R^{2}=0.999$ for $k_{1}=254 \pm 5$, $\tau_{1}=9.6 \pm 1.5, \quad k_{2}=917 \pm 39, \tau_{2}=1.19 \pm 0.06$ and $\Theta$ (the asymptotic core genome size $)=967 \pm 14$. A single exponential decay fit to core genome size data for $N \geqslant 5$ (the last ten points) resulted in a similar estimate of $\Theta$, indicating that the double exponential decay captures correctly the asymptotic trend of the core genome.

The regression analysis for new genes and for the pan-genome was performed according to a previously described method (Tettelin et al., 2008 ). The power laws $n_{\text {new }}=k_{\text {new }} N^{-\alpha}$ and $n_{\text {pan }}=k_{\text {pan }} N^{\beta}$ (Heaps' law) were fitted for $N \geqslant 5$ to new gene and pan-genome data, respectively, with a weighted least square regression, where $n_{\text {new }}$ and $n_{\text {pan }}$ are the average of new gene and pan-genome distributions, respectively, $N$ is the number of genomes, $k_{\text {new }}, k_{\text {pan }}, \alpha$ and $\beta$ are free parameters, and the inverse square of the respective distributions are weights. The best fits were obtained for $k_{\text {new }}=808 \pm 7, \alpha=0.64 \pm 0.01$ $\left(R^{2}=0.999\right)$ and for $k_{\mathrm{pan}}=1797 \pm 21, \quad \beta=0.40 \pm 0.01 \quad\left(R^{2}=0.999\right)$. Values of $\alpha>1$ and $\beta>0$ both indicate an open pan-genome. Of note, the values obtained for the pan-genome exponents $\alpha$ and $\beta$ are in good agreement with the theoretical prediction $\alpha=1-\beta$, and values obtained performing the same regressions for $N \geqslant 4,6,7,8$ were all in complete agreement with those reported, supporting the solidity of the result for the asymptotic tendency of this sample.

An open pan-genome of a specific bacterial group indicates that genomes of such taxa are evolving and diversify at a higher rate compared with a closed pan-genome.

Proteome comparison and extraction of shared and unique genes. Each of the nine predicted bifidobacterial proteomes was searched for orthologues against the other proteome, where orthology between two proteins was defined as the best bidirectional FASTA hits (Pearson, 2000).
Identification of orthologues, paralogues and unique genes was performed following a preliminary step consisting of the comparison of each protein against all other proteins using BLAST analysis (Altschul et al., 1990) (cut-off: $E$-value $1 \times 10^{-4}$ and $30 \%$ identity over at least $80 \%$ of both protein sequences), then all proteins were clustered into protein families using MCL (graph-theory-based Markov clustering algorithm) (van Dongen, 2000). Following this, the identified paralogues among the nine bifidobacterial proteomes were discarded and through MCL the unique proteins for each species of the genus Bifidobacterium were classified. Finally, each unique protein sequence, whose combined coding sequences form the core genome, was functionally annotated according to a COG category assignment.

Whole-genome alignments at DNA and protein level. Bifidobacterium whole-genome sequence alignments were performed at DNA level using MUMmer (Kurtz et al., 2004).

Comparisons at protein level (Canchaya et al., 2006; Chan et al., 2006) were performed with the PROmer algorithm (part of the MUMmer package). Distances between the nine bifidobacterial genomes were calculated from the PROmer alignment normalized by the size of the shortest genome and were compared by using the following equation:

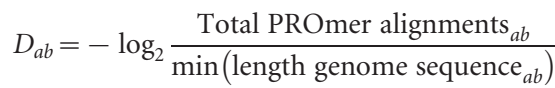

Proteome comparison and identification of orthologues. Proteomes of the currently complete actinobacterial genomes available in public databases were compared firstly using an allagainst-all BLAST (Altschul et al., 1990) (cut-off: E-value $1 \times 10^{-4}$ and $30 \%$ identity over at least $80 \%$ of both protein sequences), then the 
BLAST results were clustered into proteins families using MCL (van Dongen, 2000). Paralogues were discarded by selecting the families that contained one single protein member for each genome. Proteins identified as belonging to the genome mobilome, such as IS elements or phages, were discarded. Orthologues were functionally classified using COG category assignments.

Each set of orthologous proteins were aligned using CLUSTAL_W (Thompson et al., 2002) and phylogenetic trees were constructed using the maximum-likelihood in PhyML (Guindon \& Gascuel, 2003). The supertree was built using SplitsTree (Huson, 1998).

165 rRNA-based phylogenetic tree. A complete phylogenetic analysis was performed using the $16 \mathrm{~S}$ rRNA actinobacterial sequences retrieved from the Ribosomal Database Project-II (Cole et al., 2005). Where available, $16 \mathrm{~S}$ rRNA gene sequences of the same strain used in the proteome comparison were chosen for each species. Otherwise, the $16 \mathrm{~S}$ rRNA gene sequence of the type strain was used. Sequences were aligned using CLUSTAL_W (Thompson et al., 2002), and phylogenetic trees were constructed using the maximum-likelihood in PhyML (Guindon \& Gascuel, 2003) and neighbour-joining in CLUSTAL _ W. Furthermore, bootstrap analysis was performed using 100 resamplings.

\begin{abstract}
Identification of shared genes between bifidobacteria and other human gut microbiota members. Proteins encoded by the nine bifidobacterial strains isolated from various sections of the human GIT, such as B. adolescentis ATCC15703, B. dentium Bd1, B. longum subsp. infantis ATCC15697, B. longum subsp. longum NCC2705 and B. longum subsp. longum DJO10A, B. longum subsp. longum JDM301, Bifidobacterium animalis subsp. lactis AD011, B. animalis subsp. lactis Bl-04 and B. animalis subsp. lactis DSM10140, were compared with the proteins encoded by the genomes of 47 bacterial and two archaeal inhabitants of the human GIT or bacteria isolated from other environments (soil, water and plant) whose genomes were used as outgroups (Supplementary Table S1, available with the online version of this paper). The set of clustered genes, which had been obtained using the MCL analysis (van Dongen, 2000) as described above, was filtered in order to only retain hits that correspond to genes shared by some but not all of the analysed genomes. Furthermore, a binary matrix with vectors consisting of $0-1$ values was built by an internal Visual Basic script on a Microsoft Excel table. In this matrix, each vector corresponds to an organism, and the 0 or 1 values indicate absence or presence, respectively, of a particular gene in that organism. The final matrix $D$ represents the genome information of 55 organisms and includes data derived from the analysis of more than 11000 proteins.
\end{abstract}

Multivariate analysis. A correspondence analysis was carried out on this final matrix D using the statistical tool R 2.9.1 (Dessau \& Pipper, $2008)$; the product between the transpose of the matrix $D\left(D^{\mathrm{T}}\right.$ with 11000 rows and 55 columns) and the matrix $D$ itself (55 rows and $>11000$ columns) generates the matrix $E$ (11000 rows and 55 columns). Eigenvectors and eigenvalues of the matrix $E$ were calculated with the EIGEN subroutine from the statistical tool R 2.9.1 (Dessau \& Pipper, 2008). Only the first 10 eigenvectors were taken into consideration, thus generating matrix $F$ (11000 rows and 10 columns). The product between the matrix $D$ and matrix $F$ generated matrix $G$ (55 rows and 10 columns), which provides the position coordinates of the 55 bacteria in the first 10 axes of ordination. The percentage variation fraction associated with each axis was calculated as the ratio of the corresponding eigenvalue to the sum of all eigenvalues. Finally, the evolutionary relationships between organisms were visualized by the construction of a bidimensional plot with the statistical tool SPSS 9.0.

The information carried by matrix $F$ reveals ORFs that maximally contribute to the clustering of 55 bacterial species under examination at each axis of ordination. The ORFs corresponding to the highest values of this matrix, and which are thus of most phylogenetic relevance, were selected and evaluated with a $\chi^{2}$ test.

\section{RESULTS AND DISCUSSION}

\section{Whole-genome alignments}

Sequence alignment of the nine publicly available complete bifidobacterial genomes was performed in order to establish the genetic relationships among these members of the Bifidobacterium genus. The closest alignments that were obtained for bifidobacteria include those between members of the $B$. longum phylogenetic species, i.e. $B$. longum subsp. longum and $B$. longum subsp. infantis, or species belonging to the $B$. adolescentis group such as $B$. adolescentis versus $B$. dentium (data not shown). In contrast, the least related genomes, as concluded from their low levels of alignment, were those of $B$. animalis subsp. lactis (B. pseudolongum group) and genomes from members of the $B$. longum group, a finding that correlates well with the evolutionary distance between these species based on 16S rRNA or multigene alignments (Ventura et al., 2006).

Some of the bifidobacterial whole-genome alignments display as patched regions arranged in an X-shaped distribution across the origin of replication, a finding which was previously described for bacteria (Eisen et al., 2000). Such symmetry indicates that paring of sequences tends to occur at the same side as the origin, which is explained by the fork replication theory (Tillier \& Collins, 2000). However, these $X$-shaped regions are not visible in any of the $B$. animalis alignments. Furthermore, these Xshaped alignments can be observed most clearly in the middle region of the $x y$ plots. In fact, the rightmost and leftmost parts of the plots that correspond to the ori do not display synteny, suggesting that such regions are subject to a higher rate of reshuffling or an increased acquisition of alien DNA by horizontal gene transfer (HGT) compared with the alignment within the $B$. longum phylogenetic group. Such findings contrast with what has previously been described for bacterial genome evolution, where it was suggested that the regions around the replication terminus display an increased frequency of HGT compared with the remainder of the chromosome (Daubin \& Perriere, 2003).

These results clearly show that genomes of different bifidobacterial species are in general not collinear, thus implying the existence of significant genome diversity within members of the genus Bifidobacterium. The genome alignments also generate data that can be used to reconstruct phylogenetic relationships between bifidobacteria through the use of PROmer, similar to what was previously described for other prokaryotic genomes (Henz et al., 2005). A neighbour-joining tree was built using the distances calculated using the PROmer analyses (Fig. 1a). The resulting tree, as based on the deduced proteomes, shows a clustering of bifidobacterial species which is very 


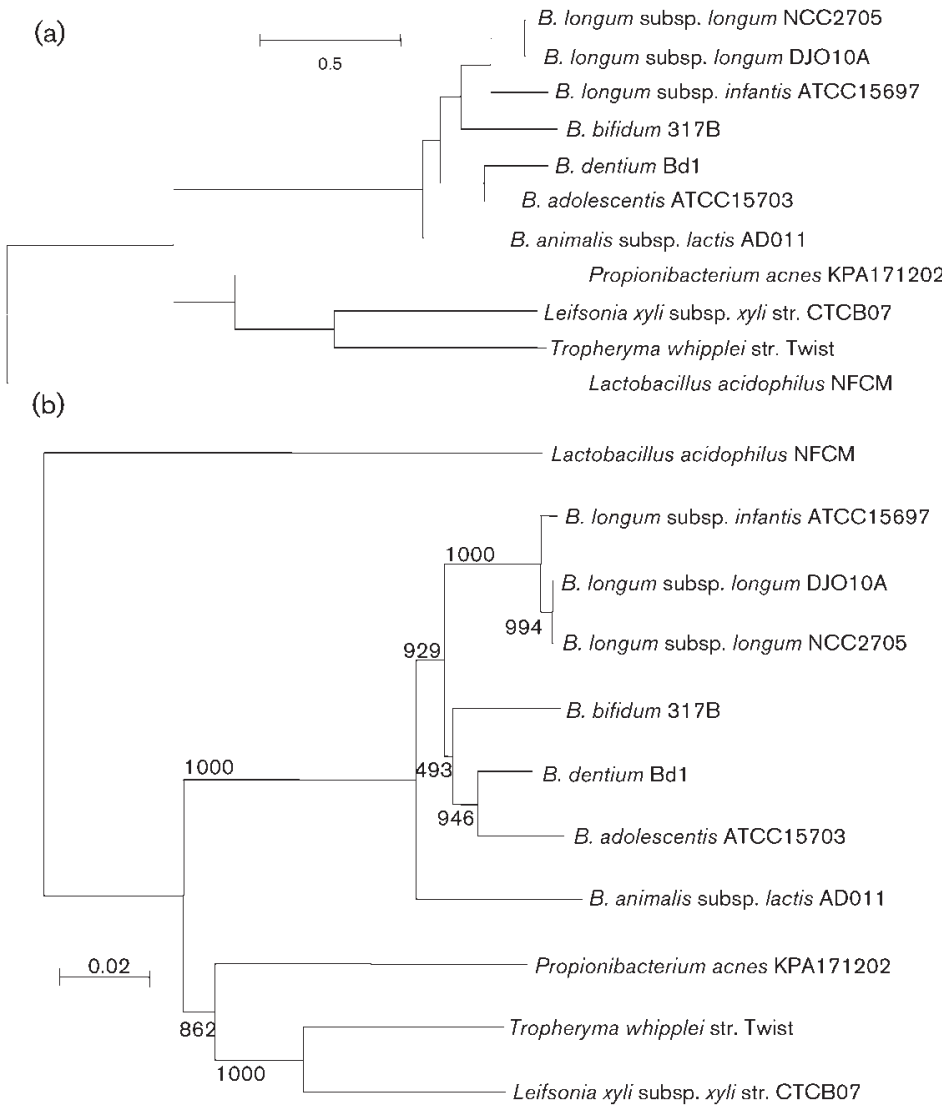

Fig. 1. Neighbour-joining tree of sequenced bifidobacteria based on PROmer distances (a). The tree is based on the distances evaluated from the non-overlapping protein aligned regions. (b) Phylogenetic tree of bifidobacteria based on 16S rRNA gene sequences. Bootstraping values are provided. Bars, number of substitutions per site.

similar to that obtained from $16 \mathrm{~S}$ rRNA comparative analysis (Fig. 1b) or from a multigene approach (a so-called supertree, Ventura et al., 2006). Thus, we can argue that in the case of bifidobacteria, whole-genome-based phylogenetic investigations, also called phylogenomics, generate a very similar evolutionary pattern to that achieved using classical molecular markers. Such a phylogenomics-based analysis provides convincing proof that all bifidobacteria share a common ancestor, and in order to expand on this finding, a more in depth analysis involving the identification of a common bifidobacterial gene content was performed.

\section{The Bifidobacterium conserved core genome}

Analysis of the set of genes/proteins that were separated from the last common ancestor, in an evolutionary time-frame, by a speciation event (Fitch, 1970) allowed the identification of 506 orthologues shared by all nine species of the genus Bifidobacterium for which the complete genome sequence was available (data not shown). This common set of proteins represents the presumed core of bifidobacterial genomic coding sequences. To estimate an asymptotic limit to the number of genes present in every Bifidobacterium species (Bifidobacterium core genome), we included in our analysis five publicly available but incomplete genomic sequences, adding to the sample five novel species of the genus: $B$. pseudocatenulatum, B. gallicum, B. catenulatum, B. bifidum and $B$. angulatum. The number of shared genes found on sequential addition of each new bifidobacterial genome sequence was extrapolated by fitting an exponential decaying function to core genes calculated with all the possible permutations in the order of addition of genome sequences (Fig. 2a). As expected, the number of shared genes initially decreases with the addition of each new genome sequence. Nevertheless, extrapolation of the curve shows that the core genome content reaches a minimum of approximately 967 genes, as indicated in Fig. 2(a). This apparent difference from the number of orthologous proteins shared between all the bifidobacterial genomes (506 proteins) is probably a consequence of the reduced stringency that is normally employed for the prediction of the core genome sequences (50\% similarities over $50 \%$ of the total gene nucleotide length) (Medini et al., 2005; Tettelin et al., 2005; Tettelin et al., 2008), compared with the higher constraint values that are usually employed in the determination of common orthologous protein sets in bacterial genomes (cut-off $E$ value $1 \times 10^{-4}$ with an identity value of $30 \%$ over $80 \%$ of both protein sequences). Of note, the actual number of shared genes in each genome varies because of duplicated genes and paralogues. Examination of the functional annotation of these genes suggests that the conserved core genes encode mostly core housekeeping functions such as those involved in replication, transcription and translation, as well as functions related to adaptation/interaction with a particular environment, such as carbohydrate metabolism, cell envelope biogenesis and signal transduction (Table 2). 


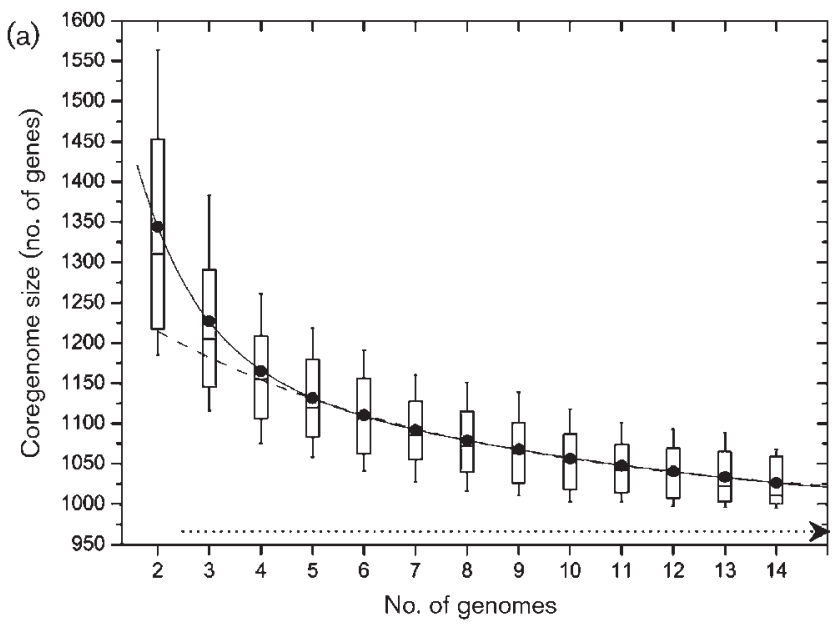

Table 2. Distribution of the genes constituting the core genome sequences of bifidobacteria with respect to $\mathrm{COG}$ categories

\begin{tabular}{|ll|}
\hline $\begin{array}{l}\text { Proportion of core } \\
\text { genome (\%) }\end{array}$ & COG category \\
\hline 19.2 & {$[\mathrm{~J}]$ Translation, ribosomal structure and } \\
& biogenesis \\
& {$[\mathrm{K}]$ Transcription } \\
7.9 & {$[\mathrm{~L}]$ Replication, recombination and repair } \\
1.9 & {$[\mathrm{D}]$ Cell cycle control, cell division, } \\
& chromosome partitioning \\
0.3 & {$[\mathrm{~V}]$ Defence mechanisms } \\
3.9 & {$[\mathrm{~T}]$ Signal transduction mechanisms } \\
4.6 & {$[\mathrm{M}]$ Cell wall/membrane/envelope } \\
& biogenesis \\
0.7 & {$[\mathrm{U}]$ Intracellular trafficking, secretion and } \\
& vesicular transport \\
4.3 & {$[\mathrm{O}]$ Post-translational modification, protein } \\
& turnover, chaperones \\
4.8 & {$[\mathrm{C}]$ Energy production and conversion } \\
6.5 & {$[\mathrm{G}]$ Carbohydrate transport and metabolism } \\
12.2 & {$[\mathrm{E}]$ Amino acid transport and metabolism } \\
6.0 & {$[\mathrm{~F}]$ Nucleotide transport and metabolism } \\
3.9 & {$[\mathrm{H}]$ Coenzyme transport and metabolism } \\
2.6 & {$[\mathrm{I}]$ Lipid transport and metabolism } \\
2.9 & {$[\mathrm{P}]$ Inorganic ion transport and metabolism } \\
0.2 & {$[\mathrm{Q}]$ Secondary metabolites biosynthesis, } \\
& transport and catabolism \\
8.4 & {$[\mathrm{R}]$ General function prediction only } \\
5.7 & {$[\mathrm{~S}]$ Function unknown } \\
&
\end{tabular}

Fig. 2. The distributions of the number $(n)$ of core genes (a), total genes (i.e. pan-genome size; b) and new genes (c) found upon sequential addition of ' $N$ ' genomes (see Methods) are shown as box-plots indicating 25-75 percentiles, the medians as horizontal lines, the means as black circles and the whiskers for 10-90 percentiles. In (a), a double exponential regression to core genome data is shown as a solid curve, a single exponential regression to $N \geqslant 5$ data is shown as a dashed curve, and the asymptotic value of the core genome obtained ( $\Theta=967 \pm 14$ genes) is indicated by a dotted arrow. In (b) and (c), power law regressions to the pangenome size and to the number of new genes are shown as solid curves, both indicating an open pan-genome (power law exponents $\beta=0.40 \pm 0.01$ and $\alpha=0.64 \pm 0.01$ ). For comparison, the limiting trend for an open/closed pan-genome (logarithmic divergence) is shown as a dashed curve in both panels.

\section{The Bifidobacterium pan-genome}

In order to estimate the total gene repertoire of members of the genus Bifidobacterium (Bifidobacterium pan-genome) we employed a previously described method (Tettelin et al., 2008) which calculates both the overall number of genes discovered and the expected number of new genes contributed by each additional genomic sequence, using the same permutation scheme employed in the analysis of

core genes. The total number of genes identified when all 14 genomes are compared is 5125 (ranging between 4960 and 5309 depending on the genome permutation order), more than twice the average number of genes found in a single Bifidobacterium genome (Fig. 2b). The pan-genome size, when plotted on a log-log scale versus the number of genomes, shows a clear linear trend in agreement with the Heaps' law pan-genome model (Tettelin et al., 2008), and a robust fit of the data was obtained for an increasing power law with positive exponent $\beta=0.40$. This indicates an open Bifidobacterium pan-genome, even though within a single species sampled with two or more isolates, very little genomic variation is observed.

The number of new genes discovered by sequential addition of genome sequences is shown in Fig. 2(c). On average, each isolate contributes 521 new genes compared with a different genome (number of genomes $N=2$ ), and this number decreases as more isolates are added. The last genome $(N=14)$ contributes on average 152 new genes compared with the other 13 , a sizeable amount considering that a significant proportion of the within-species comparisons result in the discovery of no new genes. In fact, for each fixed $\mathrm{N}$, the distributions of the genome-order permutations for new genes (data not shown) are composed of three modes: i) within-species comparisons (very low number of new 
genes), ii) comparisons between isolates belonging to very distant species (high number of new genes), and iii) a major intermediate mode accounting for $\sim 65 \%$ of the comparisons, which determines the mean of the distribution, and represents the typical between-species variation observed in the Bifidobacterium genus. A power law regression to the last ten points of the dataset $(N \geqslant 5)$ confirms a behaviour in agreement with the Heaps' law predictions for an open pangenome (exponent of the power law $\alpha=0.64$ ) and suggests that the critical contribution to the pan-genome of the genus comes from between-species diversity. When we repeated such analyses focussing on specific Bifidobacterium species for which multiple strain genome sequences are available, such as $B$. animalis subsp. lactis, different results were achieved. In fact, in the case of B. animalis subsp. lactis, the number of specific genes added to the pan-genome dropped to zero after the addition of the third strain. This result probably reflects the fact that $B$. animalis subsp. lactis is a highly clonal, recently evolved taxon from the $B$. animalis species in which genome variability is associated only with very reduced intergenic sequence variation or limited to clustered regularly interspaced short palindromic repeats (Barrangou et al., 2009). Alternatively, the sequenced strains may belong to the same evolutionary clade and may not adequately represent the diversity that exists within strains belonging to the $B$. animalis subsp. lactis species.

\section{Identification of unique genes}

The pan-genome analysis, restricted to the nine closed genomes, also allowed the identification of truly unique genes (TUGs), i.e. genes present only in a reference genome and absent in any of the other bifidobacterial genomes. Such analyses were carried out as described previously (Rasko et al., 2008). The number of TUGs varies from 21 to 230 in the nine genomes analysed. The mean number of TUGs found in the Bifidobacterium genome dataset is $143 \pm 133$. The large deviation from the mean is indicative of a high degree of genome diversity in the genus Bifidobacterium. As expected, the large majority of TUGs do not have a functional annotation, and thus they may represent novel biosynthetic or human gut commensal interaction features. Interestingly, the B. longum subsp. infantis ATCC15697 genome contains the largest number of TUGs (Supplementary Table S2 and Supplementary Fig. S1). Such a finding was anticipated, as the genome sequence of this strain was shown to exhibit a significant level of diversity, perhaps due to the presence of RexAB,

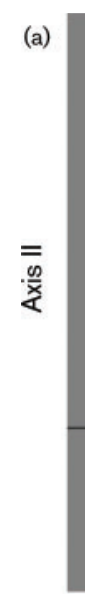

(c)

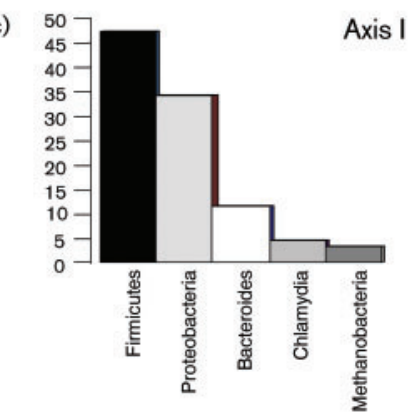

(b)

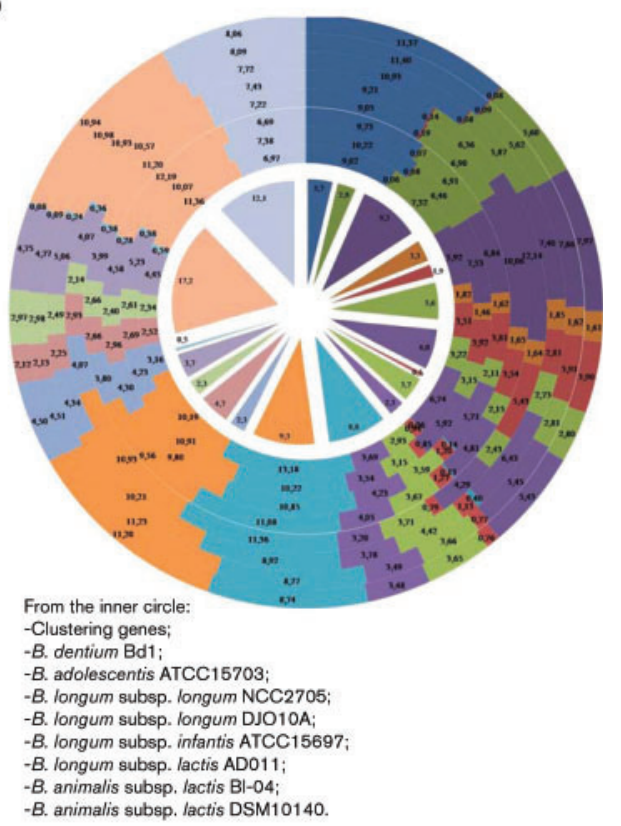

Fig. 3. (a) Plot representation of shared bifidobacterial genes versus those from intestinal human bacteria using double correspondence analysis. (b) Distribution of the shared bifidobacterial genes versus those from intestinal human bacteria according to COG categories. Each pillar represents the COG distribution in each bifidobacterial genome. (c) Schematic representation of the shared bifidobacterial genes versus those from intestinal human bacteria according to the bacterial groups to which they belong. In (a), the different bacterial groups are represented with different colours; bifidobacteria are circled. 
which promotes chromosomal rearrangements and integration of heterologous sequences through recombination (Sela et al., 2008). The identification of TUGs in bifidobacteria may serve to identify targets for further functional studies on specialized adaptive abilities, particularly with regard to host interactions and metabolism of host diet components. Additional functional genomic studies (e.g. whole transcriptional/proteomic profiling and gene inactivation) will be important to understand the function of this large set of unique hypothetical proteins, considering the relevance of bifidobacteria in human health, as they could represent putative biomarkers of a 'healthy' gut microbiota.

Many of the TUGs are flanked by insertion elements and display significant divergence from the average GC genome content or atypical codon usage, suggesting acquisition through HGT. Among their encoded products, putative adhesion-mediating proteins similar to fimbrial subunits and sugar metabolizing enzymes/transporters are found. As in the case of other bacterial genomes (Canchaya et al., 2003), phage-associated genes account for less than $1 \%$ of all TUGs in bifidobacteria (data not shown).

\section{Dispensable bifidobacterial genome and the human gut microbiome}

Bifidobacteria are considered to represent a significant component of the intestinal microbiota of mammals (Turroni et al., 2009a, 2009b; Ventura et al., 2007). The initial establishment of bifidobacteria in the human large intestine is believed to occur within the first couple of hours following birth, and following this they may remain present during the remainder of the host's life. In their natural environment, bifidobacteria come into contact with other bacterial components of the intestinal microbiota, with

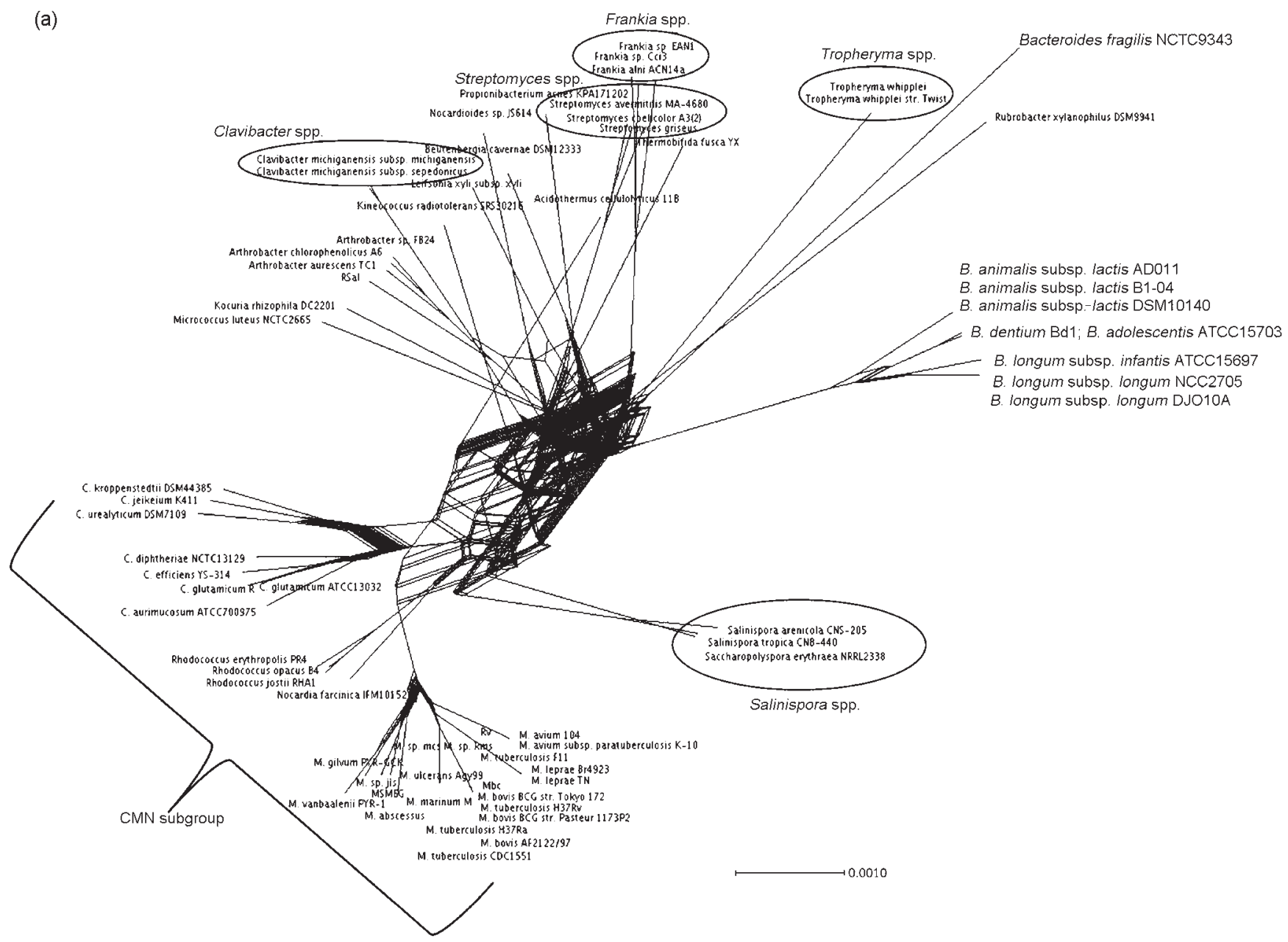

Fig. 4. (a) Phylogenetic supertree based on the sequences of actinobacterial core proteins. The various sequenced bifidobacteria are indicated; the CMN subgroup (Corynebacteria, Mycobacteria and Nocardia) is also highlighted. (b) Phylogenetic tree based on 16S rRNA gene sequences from the same actinobacterial set of species used to build the phylogenetic supertree. 
which they are expected to exchange genetic material through HGT. In order to investigate this presumption, we analysed the number of orthologues that are shared between bifidobacteria and other human gut bacteria (Supplementary Table S1). We included in our analysis those human gut bacteria whose genomes are fully sequenced, which span a broad range of taxonomic groups, i.e. from Archaea to Bacteria. These sequences were retrieved from databases and various human gut microbiome projects, and we excluded from our analyses those orthologues that are common to all bifidobacterial genomes and thus constitute the core genome sequences. The correspondence analysis (see Methods) identified 365 genes that appear to represent dispensable bifidobacterial genome sequences, i.e. genes that are shared with other bacterial members of the human gut microbiome, while they do not occur in all bifidobacterial genomes (Fig. 3a and Supplementary Table S3). When the encoded proteins were classified according to their COG categories and compared with the average COG distribution in bifidobacterial genomes, we found that, when proteins involved in metabolism and transport of carbohydrates and amino acids were excluded, the majority of the proteins were involved in coenzyme transport and metabolism, signal transduction and unknown functions (Fig. 3b). It seems logical that proteins shared between human gut bacteria contribute to adaptations that typify specific groups of intestinal commensals, emphasizing the necessity for functional investigations in order to provide the biological role of these gene products in the human gut microbiota. Interestingly, members of the human gut microbiota that appear to have exchanged genes with bifidobacteria appear to encompass a wide phylogenetic distribution including Archaea, such as Methanobrevibacterium smithii and Methanosphaera stadtmanae, as well as various taxa belonging to the phyla Chlamydiae, Proteobacteria and Firmicutes (Fig. 3c and Supplementary Table S4).

\section{Bifidobacterium phylogeny and phylogenomics}

To complement the sequence-based and synteny-based phylogenetic investigations, we also followed a multigenic tree phylogeny reconstruction approach (Fig. 4). In fact,

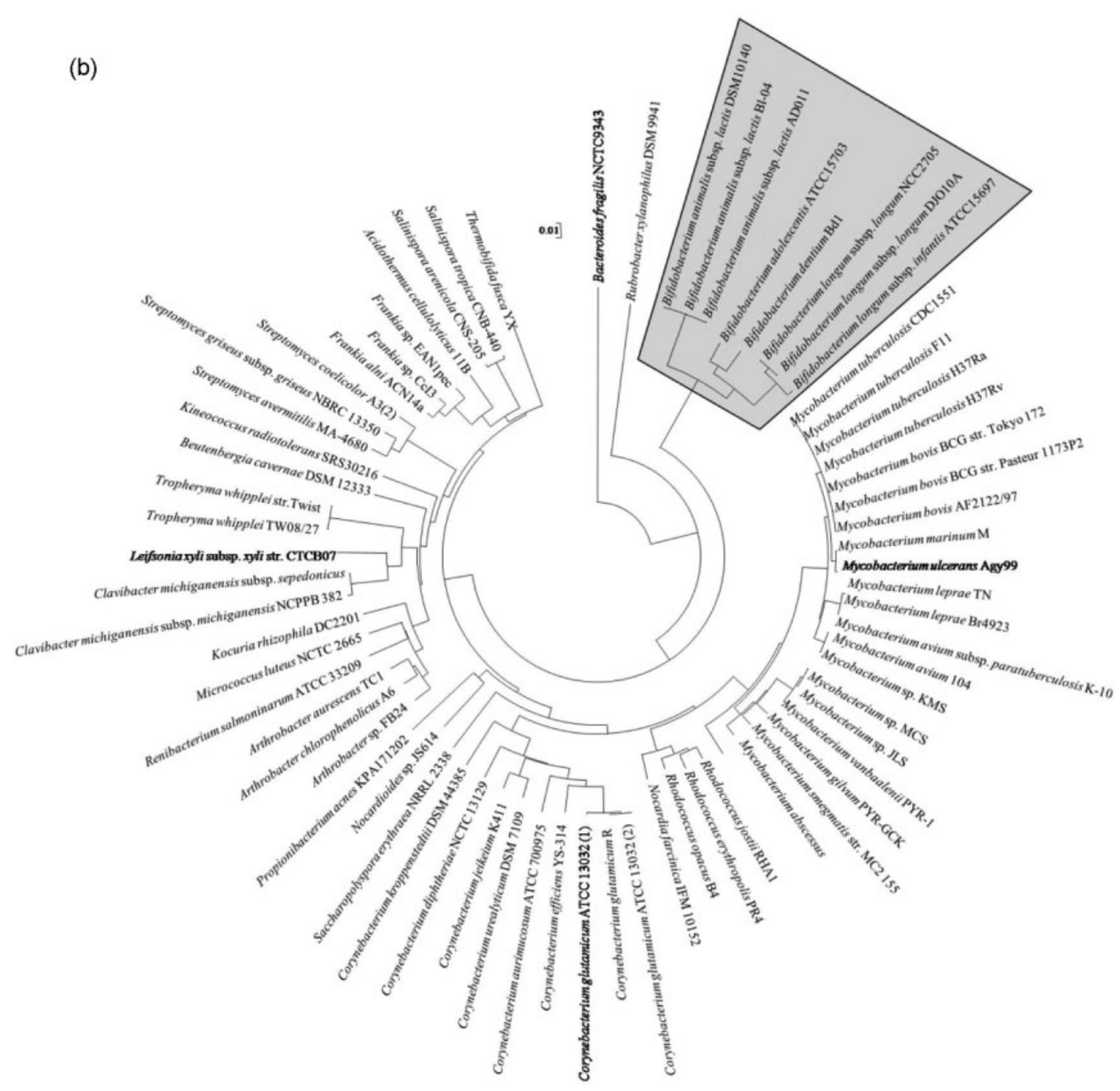


such a strategy provides a solution to the problem of combining evidence from different genome loci to infer phylogeny without losing information from independent gene histories. A concatenated protein consisting of 506 sequences, corresponding to each of the core proteins that were identified in one of the previous sections, was used to build a Bifidobacterium supertree, which allows tracing phylogeny based on genomic data (phylogenomics) within this group of bacteria. The discriminatory power of the concatenated tree is much higher than that observed with the single 16S rRNA gene, which is also confirmed by the analysis of the pairwise distances and the standard deviation, respectively. Each bifidobacterial species was differentiated as a distinct entity. The average similarity from these concatenated sequences was $74 \%$, against $95 \%$ when the $16 \mathrm{~S}$ rRNA gene was used. The most closely related taxa exhibited similarity levels of more than $90 \%$, i.e. $92 \%$ for B. longum subsp. longum and $B$. longum subsp. infantis, which are lower than those observed for the same set of strains using $16 \mathrm{~S}$ rRNA gene sequences (99\%). Furthermore, the increase in sequence size led to a considerable increase in tree robustness. In fact, the progressive concatenation showed an increase in deep-node bootstrap values. Data concatenation therefore provides a good means of increasing the robustness of the final tree. The significant increase in bootstrap values demonstrates that the phylogenetic tree, as calculated from the concatenation of core proteins that may be used as alternative molecular markers to the 16S rRNA gene, may considerably improve the phylogenetic relevance. We also performed a phylogenetic investigation based on the concatenation of a specific set of core protein sequences from both bifidobacteria and other members of the genus Actinobacteria (Supplementary Table S5). The protein set used for this purpose consisted of six housekeeping genes, $m f \mathcal{c}, r p m A$, atp $C$, obgE, ileS and $\operatorname{leg} A$, which encode translation initiation factor IF-3, 50S ribosomal protein, ATP synthase C chain, GTPase, isoleucyl-tRNA synthase and GTP-binding protein, respectively. This phylogenomics approach allowed the evolutionary positioning of bifidobacteria within the phylum Actinobacteria (Fig. 4a), which revealed that bifidobacteria represent the deepest branch separating them from other Actinobacteria in a manner that is similar to that observed for a $16 \mathrm{~S}$ rRNA-based tree (Fig. 4b).

\section{Conclusions}

The genomic era for bifidobacteria has only recently commenced in earnest, and the limited number of species for which genome sequences have been completed has allowed us to take a first look, to our knowledge, at the pan-genomic structure of this bacterial genus.

Regression analysis shows that, in the case of bifidobacteria, single species tend to be closed and only a very small number of new genes are expected to be added to the species gene pool with the addition of new genome sequences. Conversely, the size of the gene pool pertaining to the whole genus is more than twice the size of a single genome, and the pan-genome of the genus Bifidobacterium is found to be open. The analysis of nine different species in this work indicates that a substantial amount of undiscovered genes are likely to be present in the genomic sequences of isolates belonging to other, as yet unsequenced, bifidobacterial species. Such a predicted diversity is not surprising if one considers that sequencing of environmental samples, such as water, soil or faecal samples, has enabled the identification of unknown bacterial microbiomes (Eckburg et al., 2005; Venter et al., 2004). These extensive genetic datasets have indicated that the environmental gene pool available for inclusion by mechanisms such as HGT, transduction, conjugation and transformation is much larger than previously imagined. Bacteriophages are thought to play a key role in transferring genetic material between bacteria that share the same ecological niche. In contrast, bacterial species residing in restricted environments and lacking mechanisms of gene exchange may have evolved with considerably less genome variation. Bacteria such as Buchnera aphidicola or Bacillus anthracis possess a closed pangenome, where no or very limited chromosome rearrangements or gene acquisitions have occurred during the course of evolution (Tamas et al., 2002).

The current bacterial taxonomy relies on genes associated with the core genome (e.g. rRNA genes, chaperoneencoding genes, recA gene) (Stackebrandt et al., 2002). However, a large proportion of the genetic traits that are responsible for the adaptation to a specific ecological niche, such as antibiotic resistance, are presumed to be part of the dispensable genome. Therefore, sequencing of multiple strains is necessary in order to understand the genetics of adaptative strategies employed by particular commensal micro-organisms in the human gut, and to provide a more consistent definition of the species itself. In this report we show that sequencing of only a few genomes of a limited number of bifidobacterial species is not enough to understand the overall genomic basis and plasticity of the bifidobacterial genus. Also, our results suggest that in order to avoid a phylogenetic bias within this genus, further sequencing efforts should concentrate on increasing the number of species sampled rather than the number of isolates sampled per species. Alternative genome analysis techniques such as comparative genome hybridization can only provide information on the presence/absence and variability of genetic loci that are already known and do not identify genes that are not present in the reference genome. Thus, in order to fully explore the genome variability of bifidobacteria - including the identification of TUGs, the size of which could be vastly larger than the core genome - a whole genome sequencing approach will be necessary.

\section{ACKNOWLEDGEMENTS}

This work was financially supported by the Italian Award for Outstanding Young Researcher scheme 'Incentivazione alla mobilità di studiosi stranieri e italiani residenti all'estero' 2005-2009 and a Marie Curie Reintegration Grant (MERG-CT-2005-03080) to M.V., by Spinner 2013, Regione Emilia Romagna and the European Fund 
for Southeastern Europe. This work was also financially supported by an Irish Research Council for Science, Engineering and Technology Embark postgraduate fellowship to F. B. D.V.S. is a member of the Alimentary Pharmabiotic Centre, which is a Centre for Science and Technology funded by Science Foundation Ireland, through the Irish Government's National Development Plan. We thank all students and co-workers for contributing data and for their enthusiasm.

\section{REFERENCES}

Altschul, S. F., Gish, W., Miller, W., Myers, E. W. \& Lipman, D. J. (1990). Basic local alignment search tool. J Mol Biol 215, 403-410.

Alvarez-Martín, P., Florez, A. B. \& Mayo, B. (2007). Screening for plasmids among human bifidobacteria species: sequencing and analysis of pBC1 from Bifidobacterium catenulatum L48. Plasmid 57, 165-174.

Barrangou, R., Briczinski, E. P., Traeger, L. L., Loquasto, J. R., Richards, M., Horvath, P., Coûté-Monvoisin, A. C., Leyer, G., Rendulic, S. \& other authors (2009). Comparison of the complete genome sequences of Bifidobacterium animalis subsp. lactis DSM 10140 and Bl-04. J Bacteriol 191, 4144-4151.

Bentley, S. (2009). Sequencing the species pan-genome. Nat Rev Microbiol 7, 258-259.

Canchaya, C., Proux, C., Fournous, G., Bruttin, A. \& Brussow, H. (2003). Prophage genomics. Microbiol Mol Biol Rev 67, 238-276.

Canchaya, C., Claesson, M. J., Fitzgerald, G. F., van Sinderen, D. \& O'Toole, P. W. (2006). Diversity of the genus Lactobacillus revealed by comparative genomics of five species. Microbiology 152, 3185-3196.

Candela, M., Vitali, B., Matteuzzi, D. \& Brigidi, P. (2004). Evaluation of the rrn operon copy number in Bifidobacterium using real-time PCR. Lett Appl Microbiol 38, 229-232.

Chan, C. X., Beiko, R. G. \& Ragan, M. A. (2006). Detecting recombination in evolving nucleotide sequences. BMC Bioinformatics 7,412 .

Cole, J. R., Chai, B., Farris, R. J., Wang, Q., Kulam, S. A., McGarrell, D. M., Garrity, G. M. \& Tiedje, J. M. (2005). The Ribosomal Database Project (RDP-II): sequences and tools for high-throughput rRNA analysis. Nucleic Acids Res 33, D294-D296.

Cronin, M., Knobel, M., O'Connell-Motherway, M., Fitzgerald, G. F. \& van Sinderen, D. (2007). Molecular dissection of a bifidobacterial replicon. Appl Environ Microbiol 73, 7858-7866.

Daubin, V. \& Perriere, G. (2003). G+C3 structuring along the genome: a common feature in prokaryotes. Mol Biol Evol 20, 471483.

Dessau, R. B. \& Pipper, C. B. (2008). "R" - project for statistical computing. Ugeskr Laeger 170, 328-330.

Eckburg, P. B., Bik, E. M., Bernstein, C. N., Purdom, E., Dethlefsen, L., Sargent, M., Gill, S. R., Nelson, K. E. \& Relman, D. A. (2005). Diversity of the human intestinal microbial flora. Science 308, 1635-1638.

Eisen, J. A., Heidelberg, J. F., White, O. \& Salzberg, S. L. (2000). Evidence for symmetric chromosomal inversions around the replication origin in bacteria. Genome Biol 1, RESEARCH0011.

Fitch, W. M. (1970). Distinguishing homologous from analogous proteins. Syst Zool 19, 99-113.

Guindon, S. \& Gascuel, O. (2003). A simple, fast, and accurate algorithm to estimate large phylogenies by maximum likelihood. Syst Biol 52, 696-704.

Henz, S. R., Huson, D. H., Auch, A. F., Nieselt-Struwe, K. \& Schuster, S. C. (2005). Whole-genome prokaryotic phylogeny. Bioinformatics 21, 2329-2335.
Huson, D. H. (1998). SplitsTree: analyzing and visualizing evolutionary data. Bioinformatics 14, 68-73.

Kim, J. F., Jeong, H., Yu, D. S., Choi, S. H., Hur, C. G., Park, M. S., Yoon, S. H., Kim, D. W., Ji, G. E. \& other authors (2009). Genome sequence of the probiotic bacterium Bifidobacterium animalis subsp. lactis AD011. J Bacteriol 191, 678-679.

Klappenbach, J. A., Dunbar, J. M. \& Schmidt, T. M. (2000). rRNA operon copy number reflects ecological strategies of bacteria. Appl Environ Microbiol 66, 1328-1333.

Kurtz, S., Phillippy, A., Delcher, A. L., Smoot, M., Shumway, M., Antonescu, C. \& Salzberg, S. L. (2004). Versatile and open software for comparing large genomes. Genome Biol 5, R12.

Lapierre, P. \& Gogarten, J. P. (2009). Estimating the size of the bacterial pan-genome. Trends Genet 25, 107-110.

Lee, J. H. \& O'Sullivan, D. J. (2006). Sequence analysis of two cryptic plasmids from Bifidobacterium longum DJO10A and construction of a shuttle cloning vector. Appl Environ Microbiol 72, 527-535.

Lee, J. H., Karamychev, V. N., Kozyavkin, S. A., Mills, D., Pavlov, A. R., Pavlova, N. V., Polouchine, N. N., Richardson, P. M., Shakhova, V. V. \& other authors (2008). Comparative genomic analysis of the gut bacterium Bifidobacterium longum reveals loci susceptible to deletion during pure culture growth. BMC Genomics 9, 247.

Makarova, K., Slesarev, A., Wolf, Y., Sorokin, A., Mirkin, B., Koonin, E., Pavlov, A., Pavlova, N., Karamychev, V. \& other authors (2006). Comparative genomics of the lactic acid bacteria. Proc Natl Acad Sci U S A 103, 15611-15616.

Marco, M. L., Pavan, S. \& Kleerebezem, M. (2006). Towards understanding molecular modes of probiotic action. Curr Opin Biotechnol 17, 204-210.

Medini, D., Donati, C., Tettelin, H., Masignani, V. \& Rappuoli, R. (2005). The microbial pan-genome. Curr Opin Genet Dev 15, 589594.

O'Hara, A. M. \& Shanahan, F. (2007). Mechanisms of action of probiotics in intestinal diseases. Scientific World Journal 7, 31-46.

Pearson, W. R. (2000). Flexible sequence similarity searching with the FASTA3 program package. Methods Mol Biol 132, 185-219.

Rasko, D. A., Rosovitz, M. J., Myers, G. S., Mongodin, E. F., Fricke, W. F., Gajer, P., Crabtree, J., Sebaihia, M., Thomson, N. R. \& other authors (2008). The pangenome structure of Escherichia coli: comparative genomic analysis of $E$. coli commensal and pathogenic isolates. J Bacteriol 190, 6881-6893.

Sangrador-Vegas, A., Stanton, C., van Sinderen, D., Fitzgerald, G. F. \& Ross, R. P. (2007). Characterization of plasmid pASV479 from Bifidobacterium pseudolongum subsp. globosum and its use for expression vector construction. Plasmid 58, 140-147.

Schell, M. A., Karmirantzou, M., Snel, B., Vilanova, D., Berger, B., Pessi, G., Zwahlen, M. C., Desiere, F., Bork, P. \& other authors (2002). The genome sequence of Bifidobacterium longum reflects its adaptation to the human gastrointestinal tract. Proc Natl Acad Sci U S A 99, 14422-14427.

Sela, D. A., Chapman, J., Adeuya, A., Kim, J. H., Chen, F., Whitehead, T. R., Lapidus, A., Rokhsar, D. S., Lebrilla, C. B. \& other authors (2008). The genome sequence of Bifidobacterium longum subsp. infantis reveals adaptations for milk utilization within the infant microbiome. Proc Natl Acad Sci U S A 105, 1896418969.

Stackebrandt, E., Frederiksen, W., Garrity, G. M., Grimont, P. A., Kämpfer, P., Maiden, M. C., Nesme, X., Rosselló-Mora, R., Swings, J. \& other authors (2002). Report of the ad hoc committee for the reevaluation of the species definition in bacteriology. Int J Syst Evol Microbiol 52, 1043-1047. 
Tamas, I., Klasson, L., Canback, B., Naslund, A. K., Eriksson, A. S., Wernegreen, J. J., Sandstrom, J. P., Moran, N. A. \& Andersson, S. G. (2002). 50 million years of genomic stasis in endosymbiotic bacteria. Science 296, 2376-2379.

Tettelin, H., Masignani, V., Cieslewicz, M. J., Donati, C., Medini, D., Ward, N. L., Angiuoli, S. V., Crabtree, J., Jones, A. L. \& other authors (2005). Genome analysis of multiple pathogenic isolates of Streptococcus agalactiae: implications for the microbial "pangenome". Proc Natl Acad Sci U S A 102, 13950-13955.

Tettelin, H., Riley, D., Cattuto, C. \& Medini, D. (2008). Comparative genomics: the bacterial pan-genome. Curr Opin Microbiol 11, 472-477.

Thompson, J. D., Gibson, T. J. \& Higgins, D. G. (2002). Multiple sequence alignment using CLUSTAL_W and CLUSTAL_X. Curr Protoc Bioinformatics Chapter 2, Unit 2.3.

Tillier, E. R. \& Collins, R. A. (2000). Genome rearrangement by replication-directed translocation. Nat Genet 26, 195-197.

Turroni, F., Foroni, E., Pizzetti, P., Giubellini, V., Ribbera, A., Merusi, P., Cagnasso, P., Bizzarri, B., de'Angelis, G. L. \& other authors (2009a). Exploring the diversity of the bifidobacterial population in the human intestinal tract. Appl Environ Microbiol 75, 1534-1545.

Turroni, F., Marchesi, J. R., Foroni, E., Gueimonde, M., Shanahan, F., Margolles, A., van Sinderen, D. \& Ventura, M. (2009b). Microbiomic analysis of the bifidobacterial population in the human distal gut. ISME J 3, 745-751. van Dongen, S. (2000). Graph clustering by flow simulation. PhD thesis, University of Utrecht, Utrecht, The Netherlands.

Venter, J. C., Remington, K., Heidelberg, J. F., Halpern, A. L., Rusch, D., Eisen, J. A., Wu, D., Paulsen, I., Nelson, K. E. \& other authors (2004). Environmental genome shotgun sequencing of the Sargasso Sea. Science 304, 66-74.

Ventura, M., Canchaya, C., Del Casale, A., Dellaglio, F., Neviani, E., Fitzgerald, G. F. \& van Sinderen, D. (2006). Analysis of bifidobacterial evolution using a multilocus approach. Int J Syst Evol Microbiol 56, 2783-2792.

Ventura, M., Canchaya, C., Tauch, A., Chandra, G., Fitzgerald, G. F., Chater, K. F. \& van Sinderen, D. (2007). Genomics of Actinobacteria: tracing the evolutionary history of an ancient phylum. Microbiol Mol Biol Rev 71, 495-548.

Ventura, M., O'Flaherty, S., Claesson, M. J., Turroni, F., Klaenhammer, T. R., van Sinderen, D. \& O'Toole, P. W. (2009a). Genome-scale analyses of health-promoting bacteria: probiogenomics. Nat Rev Microbiol 7, 61-71.

Ventura, M., Turroni, F., Zomer, A., Foroni, E., Giubellini, V., Bottacini, F., Canchaya, C., Claesson, M. J., He, F. \& other authors (2009b). The Bifidobacterium dentium $\mathrm{Bd} 1$ genome sequence reflects its genetic adaptation to the human oral cavity. PLoS Genet 5, e1000785.

Edited by: H. J. Flint 